\title{
RANDERS METRICS OF BERWALD TYPE ON 4-DIMENSIONAL HYPERCOMPLEX LIE GROUPS
}

\author{
H. R. SALIMI MOGHADDAM
}

\begin{abstract}
In the present paper we study Randers metics of Berwald type on simply connected 4-dimensional real Lie groups admitting invariant hypercomplex structure. On these spaces, the Randers metrics arising from invariant hyper-Hermitian metrics are considered. Then we give explicit formulas for computing flag curvature of these metrics. By this study we construct two 4-dimensional Berwald spaces, one of them has non-negative flag curvature and the other one has non-positive flag curvature.
\end{abstract}

\section{Introduction}

Hyper-Kähler Geometry with Torsion (HKT-geometry) is an important field in differential geometry which have many applications in theoretical physics. These structures appear in supersymmetric sigma model and the study of black holes (see [11] and [13].). The background object of HKT-geometry is hyper-Hermitian manifolds.

A special class of hyper-Hermitian manifolds are Lie groups. On Lie groups, we can consider those hypercomplex structure and those hyper-Hermitian (Riemannian) metrics which are invariant under left action of $G$ on itself. The simply connected 4-dimensional real Lie groups admitting invariant hypercomplex structure equipped with left invariant hyper-Hermitian (Riemannian) metrics classified by M. L. Barberis (see [4] and [5].). Also we completely described the Levi-Civita connection and sectional curvature of these metrics in [18].

On the other hand Finsler metrics which are a generalization of Riemannian metrics have been found many applications in physics ([1, [2]). Invariant Finsler metrics on homogeneous spaces and Lie groups are of interesting Finsler metrics which have been studied in the recent years ([7], [8], [9], [10], [15], [16] and [17]).

In this paper we study left invariant Randers metrics (a special Finsler metric) which arise from left invariant hyper-Hermitian (Riemannian) metrics and parallel left invariant vector fields on simply connected 4-dimensional real Lie groups admitting invariant hypercomplex structure. We give the explicit formula for computing flag curvature, a generalization of sectional curvature for Finsler metrics, of such Randers metrics. In this way we introduce two

Key words and phrases. hypercomplex manifold, hyper-Hermitian metric, left invariant metric, Randers metric, Berwald metric, flag curvature

AMS 2000 Mathematics Subject Classification: 53C15, 58B20, 53B35, 53C60. 
special complete Randers spaces of Berwald type, one of them has non-negative flag curvature and the other one has non-positive flag curvature.

\section{Preliminaries}

Suppose that $M$ is a $4 n$-dimensional manifold. Also let $J_{i}, i=1,2,3$, be three fiberwise endomorphism of $T M$ such that

$$
\begin{aligned}
J_{1} J_{2} & =-J_{2} J_{1}=J_{3}, \\
J_{i}^{2} & =-I d_{T M}, \quad i=1,2,3, \\
N_{i} & =0, \quad i=1,2,3,
\end{aligned}
$$

where $N_{i}$ is the Nijenhuis tensor (torsion) corresponding to $J_{i}$ defined as follows:

$$
N_{i}(X, Y)=\left[J_{i} X, J_{i} Y\right]-[X, Y]-J_{i}\left(\left[X, J_{i} Y\right]+\left[J_{i} X, Y\right]\right),
$$

for all vector fields $X, Y$ on $M$. Then the family $\mathcal{H}=\left\{J_{i}\right\}_{i=1,2,3}$ is called a hypercomplex structure on $M$.

In fact three complex structures $J_{1}, J_{2}$ and $J_{3}$ on a $4 n$-dimensional manifold $M$ form a hypercomplex structure if they satisfy in the relation 2.1 (since an almost complex structure is a complex structure if and only if it has no torsion, see [12] page 124.).

A Riemannian metric $g$ on a hypercomplex manifold $(M, \mathcal{H})$ is called hyper-Hermitian if $g\left(J_{i} X, J_{i} Y\right)=g(X, Y)$, for all vector fields $X, Y$ on $M$ and $i=1,2,3$.

A hypercomplex structure $\mathcal{H}=\left\{J_{i}\right\}_{i=1,2,3}$ on a Lie group $G$ is said to be left invariant if for any $a \in G$,

$$
J_{i}=T l_{a} \circ J_{i} \circ T l_{a^{-1}}
$$

where $T l_{a}$ is the differential function of the left translation $l_{a}$.

Let $M$ be a smooth $n$-dimensional manifold and $T M$ be its tangent bundle. A Finsler metric on $M$ is a non-negative function $F: T M \longrightarrow \mathbb{R}$ which has the following properties:

(1) $F$ is smooth on the slit tangent bundle $T M^{0}:=T M \backslash\{0\}$,

(2) $F(x, \lambda y)=\lambda F(x, y)$ for any $x \in M, y \in T_{x} M$ and $\lambda>0$,

(3) the $n \times n$ Hessian matrix $\left[g_{i j}(x, y)\right]=\left[\frac{1}{2} \frac{\partial^{2} F^{2}}{\partial y^{i} \partial y^{j}}\right]$ is positive definite at every point $(x, y) \in T M^{0}$.

In 1941, G. Randers introduced an important type of Finsler metrics by using Riemannian metrics and 1-forms on manifolds ([14]). Randers metrics are as follows:

$$
F(x, y)=\sqrt{g_{i j}(x) y^{i} y^{j}}+b_{i}(x) y^{i},
$$

where $g=\left(g_{i j}(x)\right)$ is a Riemannian metric and $b=\left(b_{i}(x)\right)$ is a nowhere zero 1 -form on $M$. It has been shown that $F$ is a Finsler metric if and only if $\|b\|=b_{i}(x) b^{i}(x)<1$, where $b^{i}(x)=g^{i j}(x) b_{j}(x)$ and $\left[g^{i j}(x)\right]$ is the inverse matrix of $\left[g_{i j}(x)\right]$. 
There is another way for describing Randers metrics. We can replace the 1 -form $b=\left(b_{i}(x)\right)$ with its dual, therefore Randers metrics can be defined as follows:

$$
F(x, y)=\sqrt{g(x)(y, y)}+g(x)(X(x), y)
$$

where $X$ is a vector field on $M$ such that $\|X\|=\sqrt{g(X, X)}<1$.

$F$ is of Berwald type if and only if $X$ is parallel with respect to the Levi-Civita connection induced by the Riemannian metric $g$ (see [3].).

A Riemannian Metric $g$ on the Lie group $G$ is called left invariant if

$$
g(x)(y, z)=g(e)\left(T_{x} l_{x^{-1}} y, T_{x} l_{x^{-1}} z\right) \quad \forall x \in G, \forall y, z \in T_{x} G,
$$

where $e$ is the unit element of $G$.

Suppose that $g$ is a left invariant Riemannian metric on a Lie group $G$ with Lie algebra $\mathfrak{g}$, then the Levi-Civita connection of $g$ is defined by the following relation

$$
2 g\left(\nabla_{U} V, W\right)=g([U, V], W)-g([V, W], U)+g([W, U], V),
$$

for any $U, V, W \in \mathfrak{g}$, where $<,>$ is the inner product induced by $g$ on $\mathfrak{g}$.

We can define left invariant Finsler metrics similar to the Riemannian case. A Finsler metric is called left invariant if

$$
F(x, y)=F\left(e, T_{x} l_{x^{-1}} y\right) .
$$

The simplest way for constructing left invariant Randers metrics on Lie groups is the use of left invariant Riemannian metrics and left invariant vector fields. Suppose that $G$ is a Lie group, $g$ is a left invariant Riemannian metric and $X$ is a left invariant vector field such that $\sqrt{g(X, X)}<1$, then we can define a left invariant Randers metric $F$ as the formula 2.7. An important quantity which associates with a Finsler space is flag curvature. This quantity is a natural generalization of the concept of sectional curvature in Riemannian geometry which is computed by the following formula:

$$
K(P, Y)=\frac{g_{Y}(R(U, Y) Y, U)}{g_{Y}(Y, Y) \cdot g_{Y}(U, U)-g_{Y}^{2}(Y, U)},
$$

where $g_{Y}(U, V)=\left.\frac{1}{2} \frac{\partial^{2}}{\partial s \partial t}\left(F^{2}(Y+s U+t V)\right)\right|_{s=t=0}, P=\operatorname{span}\{U, Y\}, R(U, Y) Y=\nabla_{U} \nabla_{Y} Y-$ $\nabla_{Y} \nabla_{U} Y-\nabla_{[U, Y]} Y$ and $\nabla$ is the Chern connection induced by $F$ (see [3] and [19].).

From now we suppose that $G$ is a simply connected 4-dimensional real Lie group.

\section{Randers Metrics of Berwald type on 4-dimensional hypercomplex Lie groups}

In this paper we consider left invariant hyper-Hermitian Riemannian metrics on left invariant hypercomplex 4-dimensional simply connected Lie groups. These spaces have been classified by M. L. Barberis as follows (for more details see [4.):

Let $G$ be a Lie group as above with Lie algebra $\mathfrak{g}$. She has shown that $g$ is either Abelian or isomorphic to one of the following Lie algebras: 
(1) $[Y, Z]=W,[Z, W]=Y,[W, Y]=Z, X$ central,

(2) $[X, Z]=X,[Y, Z]=Y,[X, W]=Y,[Y, W]=-X$,

(3) $[X, Y]=Y,[X, Z]=Z,[X, W]=W$,

(4) $[X, Y]=Y,[X, Z]=\frac{1}{2} Z,[X, W]=\frac{1}{2} W,[Z, W]=\frac{1}{2} Y$,

where $\{X, Y, Z, W\}$ is an orthonormal basis.

The case (1) is diffeomorphic to $\mathbb{R} \times S^{3}$ and the other cases are diffeomorphic to $\mathbb{R}^{4}$ (see [4] and [5].).

Now we discuss about left invariant Randers metrics of Berwald type which can arise from these Riamannian metrics and left invariant vector fields on these spaces.

We begin with Abelian case. For this case we have the following theorem (see [17.).

Theorem 3.1. Let $G$ be an abelian Lie group equipped with a left invariant Riemannian metric $g$ and let $\mathfrak{g}$ be the Lie algebra of $G$. Suppose that $X \in \mathfrak{g}$ is a left invariant vector field with $\sqrt{g(X, X)}<1$. Then the Randers metric $F$ defined by the formula 2.7 is a flat geodesically complete locally Minkowskian metric on $G$.

Now we continue with the other four cases.

Case 1. We can compute the Levi-Civita connection by using formula 2.9 (Also you can see [18].). The Levi-Civita connection is as follows:

$$
\begin{aligned}
& \nabla_{X} X=0, \quad \nabla_{X} Y=0, \quad \nabla_{X} Z=0, \quad \nabla_{X} W=0, \\
& \nabla_{Y} X=0, \quad \nabla_{Y} Y=0, \quad \nabla_{Y} Z=\frac{1}{2} W, \quad \nabla_{Y} W=-\frac{1}{2} Z, \\
& \nabla_{Z} X=0, \quad \nabla_{Z} Y=-\frac{1}{2} W, \quad \nabla_{Z} Z=0, \quad \nabla_{Z} W=\frac{1}{2} Y, \\
& \nabla_{W} X=0, \quad \nabla_{W} Y=\frac{1}{2} Z, \quad \nabla_{W} Z=-\frac{1}{2} Y, \quad \nabla_{W} W=0 .
\end{aligned}
$$

A simple computation shows that the only family of vector fields which is parallel with respect to this connection is of the form $Q=q X$ for any $q \in \mathbb{R}$. Now let $0<\|Q\|<1$ or equivalently let $0<|q|<1$, therefore by using these left invariant vector fields $Q$ and formula 2.7, $G$ admits a family of Randers metrics of Berwald type.

Case 2. The formula 2.9 shows that the Levi-Civita connection of the Riemannian metric of $G$ is of the form $([18])$ :

$$
\begin{aligned}
& \nabla_{X} X=-Z, \quad \nabla_{X} Y=0, \quad \nabla_{X} Z=X, \quad \nabla_{X} W=0 \\
& \nabla_{Y} X=0, \quad \nabla_{Y} Y=-Z, \quad \nabla_{Y} Z=Y, \quad \nabla_{Y} W=0 \\
& \nabla_{Z} X=0, \quad \nabla_{Z} Y=0, \quad \nabla_{Z} Z=0, \quad \nabla_{Z} W=0 \\
& \nabla_{W} X=-Y, \quad \nabla_{W} Y=X, \quad \nabla_{W} Z=0, \quad \nabla_{W} W=0 .
\end{aligned}
$$


Similar to case 1 by a simple computation we can show that the only parallel vector fields with respect to $\nabla$ are of the form $Q=q W, q \in \mathbb{R}$. For constructing a non-Riemannian family of Randers metrics of Berwald type defined by formula 2.7, it is sufficient to let $0<\|Q\|<1$ or equivalently $0<|q|<1$.

Case 3, and case4. The Levi-Civita connections of case 3 and 4 are of the following forms respectively:

$$
\begin{aligned}
& \nabla_{X} X=0, \quad \nabla_{X} Y=0, \quad \nabla_{X} Z=0, \quad \nabla_{X} W=0 \\
& \nabla_{Y} X=-Y, \quad \nabla_{Y} Y=X, \quad \nabla_{Y} Z=0, \quad \nabla_{Y} W=0, \\
& \nabla_{Z} X=-Z, \quad \nabla_{Z} Y=0, \quad \nabla_{Z} Z=X, \quad \nabla_{Z} W=0, \\
& \nabla_{W} X=-W, \quad \nabla_{W} Y=0, \quad \nabla_{W} Z=0, \quad \nabla_{W} W=X
\end{aligned}
$$

and

$$
\begin{aligned}
& \nabla_{X} X=0, \quad \nabla_{X} Y=0, \quad \nabla_{X} Z=0, \quad \nabla_{X} W=0, \\
& \nabla_{Y} X=-Y, \quad \nabla_{Y} Y=X, \quad \nabla_{Y} Z=-\frac{1}{4} W, \quad \nabla_{Y} W=\frac{1}{4} Z, \\
& \nabla_{Z} X=-\frac{1}{2} Z, \quad \nabla_{Z} Y=-\frac{1}{4} W, \quad \nabla_{Z} Z=\frac{1}{2} X, \quad \nabla_{Z} W=\frac{1}{4} Y, \\
& \nabla_{W} X=-\frac{1}{2} W, \quad \nabla_{W} Y=\frac{1}{4} Z, \quad \nabla_{W} Z=-\frac{1}{4} Y, \quad \nabla_{W} W=\frac{1}{2} X .
\end{aligned}
$$

It is easy to show that these connections do not admit any parallel left invariant vector field, therefore there is not any left invariant Randers metric of Berwald type arising from left invariant vector fields (by using formula 2.7) on these Riemannian Lie groups.

\section{Flag curvature}

In this section we discuss about the flag curvature of invariant Randers metrics of cases 1 and 2 .

Case 1. By using Levi-Civita connection for curvature tensor we have ([18]):

$$
\begin{aligned}
& R(Y, Z) Y=-R(Z, W) W=-\frac{1}{4} Z, \\
& R(Y, W) W=R(Y, Z) Z=\frac{1}{4} Y, \\
& R(Z, W) Z=R(Y, W) Y=-\frac{1}{4} W,
\end{aligned}
$$

and in other cases $R=0$. Now let $U=a X+b Y+c Z+d W$ and $V=\tilde{a} X+\tilde{b} Y+\tilde{c} Z+\tilde{d} W$ be two arbitrary vectors in $\mathfrak{g}$ then we have:

$$
\left(4.2 \mathcal{R}(V, U) U=-\frac{1}{4}\{(b \tilde{c}-c \tilde{b})(c Y-b Z)+(b \tilde{d}-d \tilde{b})(d Y-b W)+(c \tilde{d}-d \tilde{c})(d Z-c W)\} .\right.
$$

Since $F$ is of Berwald type therefore the curvature tensor of $F$ and $g$ coincide. Suppose that $\{U, V\}$ is an orthonormal basis for $P=\operatorname{span}\{U, V\}$ with respect to the inner product $<,>$ 
induced by $g$. Now by using formula $g_{U}\left(V_{1}, V_{2}\right)=\left.\frac{1}{2} \frac{\partial^{2}}{\partial s \partial t}\left(F^{2}\left(U+s V_{1}+t V_{2}\right)\right)\right|_{s=t=0}$ of $F$ (for an explicit formula you can see [9].) we have:

$$
\begin{aligned}
g_{U}(R(V, U) U, V) & =\frac{1}{4}(1+a q)\left\{(b \tilde{c}-c \tilde{b})^{2}+(b \tilde{d}-d \tilde{b})^{2}+(c \tilde{d}-d \tilde{c})^{2}\right\} \\
g_{U}(U, U) & =(1+a q)^{2} \\
g_{U}(V, V) & =1+a q+(\tilde{a} q)^{2} \\
g_{U}(U, V) & =\tilde{a} q(1+a q) .
\end{aligned}
$$

Now by using 2.11 we have:

$$
K(P, U)=\frac{(b \tilde{c}-c \tilde{b})^{2}+(b \tilde{d}-d \tilde{b})^{2}+(c \tilde{d}-d \tilde{c})^{2}}{4(1+a q)^{2}} \geq 0 .
$$

Therefore in the case $1(G, F)$ is of non-negative flag curvature.

Case 2. The curvature tensor of Riemannian metric (Finsler metric) of this case is of the form:

$$
\begin{aligned}
& R(X, Y) X=-R(Y, Z) Z=Y, \\
& R(X, Y) Y=R(X, Z) Z=-X, \\
& R(X, Z) X=R(Y, Z) Y=Z .
\end{aligned}
$$

In this case for any $U$ and $V$ we have:

$(4.9) R(V, U) U=-\{(a \tilde{b}-b \tilde{a})(a Y-b X)+(a \tilde{c}-c \tilde{a})(a Z-c X)+(b \tilde{c}-c \tilde{b})(b Z-c Y)\}$,

Let $P=\{U, V\}$ be as case 1 . Therefore for the Randers metric $F$ described in case 2 we have:

$$
\begin{aligned}
g_{U}(R(V, U) U, V) & =-(1+d q)\left\{(a \tilde{b}-b \tilde{a})^{2}+(a \tilde{c}-c \tilde{a})^{2}+(b \tilde{c}-c \tilde{b})^{2}\right\} \\
g_{U}(U, U) & =(1+d q)^{2} \\
g_{U}(V, V) & =1+d q+(\tilde{d} q)^{2} \\
g_{U}(U, V) & =\tilde{d} q(1+d q) .
\end{aligned}
$$

Hence for the flag curvature we have:

$$
K(P, U)=\frac{-\left\{(a \tilde{b}-b \tilde{a})^{2}+(a \tilde{c}-c \tilde{a})^{2}+(b \tilde{c}-c \tilde{b})^{2}\right\}}{(1+d q)^{2}} \leq 0,
$$

which shows that $(G, F)$ is of non-positive flag curvature.

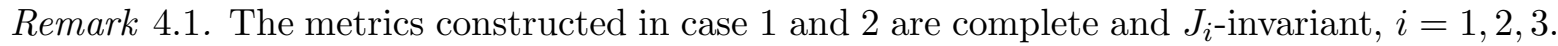

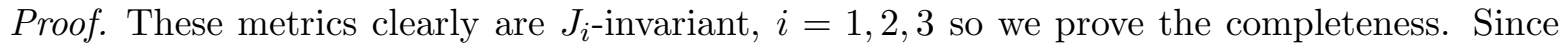
the metric $F$ (in cases 1 or 2 ) is of Berwald type therefore the geodesics of $F$ and $g$ coincide. On the other hand $(G, g)$ is a homogeneous Riemannian manifold, hence $(G, g)$ is geodesically complete (see [6] page 185.). Therefore $(G, F)$ is geodesically complete. Now the Hofp-Rinow theorem and connectedness of $G$ will complete the proof. 


\section{REFERENCES}

1. P. L. Antonelli, R. S. Ingarden, M. Matsumoto, The Theory of Sprays and Finsler Spaces with Applications in Physics and Biology, Kluwer Academic Publishers, (1993).

2. G. S. Asanov, Finsler Geometry, Relativity and Gauge Theories, D. Reidel Publishing Company, (1985).

3. D. Bao, S. S. Chern and Z. Shen, An Introduction to Riemann-Finsler Geometry, (Berlin: Springer) (2000).

4. M. L. Barberis, Hypercomplex Structures on Four-Dimensional Lie Groups, Proceeding of the American Mathematical Society 125(4), (1997) 1043-1054.

5. M. L. Barberis, Hyper-Kahler Metrics Conformal to Left Invariant Metrics on Four-Dimensional Lie Groups, Mathematical Physics, Analysis and Geometry 6, (2003) 1-8.

6. J. K. Beem, P. E. Ehrlich and K. L. Easley, Global Lorentzian Geometry, (Marcel Dekker, INC.) (1996).

7. S. Deng and Z. Hou, Invariant Finsler Metrics on Homogeneous Manifolds, J. Phys. A: Math. Gen. 37 (2004), 8245-8253.

8. S. Deng, Z. Hou, Invariant Randers Metrics on Homogeneous Riemannian Manifolds, J. Phys. A: Math. Gen. 37 (2004), 4353-4360.

9. E. Esrafilian and H. R. Salimi Moghaddam, Flag Curvature of Invariant Randers Metrics on Homogeneous Manifolds, J. Phys. A: Math. Gen. 39 (2006) 3319-3324.

10. E. Esrafilian and H. R. Salimi Moghaddam, Induced Invariant Finsler Metrics on Quotient Groups, Balkan Journal of Geometry and Its Applications, Vol. 11, No. 1 (2006) 73-79.

11. G.W. Gibbons, G. Papadopoulos and K.S. Stelle, HKT and OKT geometries on soliton black hole moduli spaces, Nucl. Phys. B 508, (1997) 623-658.

12. S. Kobayashi and K. Nomizu, Foundations of Differential Geometry, VOl.2, (INTERSCIENCE PUBLISHERS) (1969).

13. Y. S. Poon,Examples of Hyper-Kähler Connections with Torsion, Vienna, Preprint ESI 770, (1999) 1-7.

14. G. Randers, On an Asymmetrical Metric in the Four-Space of General Relativity, Phys. Rev. 59(1941), 195-199.

15. H. R. Salimi Moghaddam, On the flag curvature of invariant Randers metrics, Math. Phys. Anal. Geom. 11 (2008) 1-9.

16. H. R. Salimi Moghaddam, Flag curvature of invariant $(\alpha, \beta)$-metrics of type $\frac{(\alpha+\beta)^{2}}{\alpha}$, J. Phys. A: Math. Theor. 41 (2008).

17. H. R. Salimi Moghaddam, Some Berwald Spaces of Non-positive flag Curvature, Submitted.

18. H. R. Salimi Moghaddam, On some hypercomplex 4-dimensional Lie groups of constant scalar curvature, Submitted.

19. Z. Shen, Lectures on Finsler Geometry, (World Scientific) (2001).

Department of Mathematics, Faculty of Sciences, University of Isfahan, Isfahan,81746-73441IRAN.

E-mail address: salimi.moghaddam@gmail.com and hr.salimi@sci.ui.ac.ir 